Kai Essig, Benjamin Strenge, Thomas Schack

\title{
Die intelligente ADAMAAS-Datenbrille - Chancen und Risiken des Einsatzes mobiler Assistiver Technologien für die Inklusion
}

Zusammenfassung: Ein zentrales Ziel der Europäischen Union ist die Schaffung einer inklusiven, reflexiven und innovativen Gesellschaft. Das umfasst unter anderem die Integration von Menschen mit Behinderungen in den ersten Arbeitsmarkt, die Unterstützung von älteren Personen im beruflichen Umfeld und bei der Teilhabe in Alltagssituationen, sowie der Bereitstellung von Hilfen für Kinder und Jugendliche mit Lese-, Lern- oder Mitteilungsschwäche im Schul- und Ausbildungsbereich. Bei der Integration dieser Gruppen in das gesellschaftliche Leben können moderne, frei einsetzbare Assistive Technologien (AT) in Form von Smart Glasses, die mobil sind, nach den Bedürfnissen der Zielgruppen entwickelt wurden und sich auf die individuellen Schwächen der entsprechenden Nutzer ${ }^{1}$ einstellen können, eine gleichberechtigte und selbstbestimmte Teilhabe am Schul-, Arbeits- und Privatleben ermöglichen. Als Beispiel für ein intelligentes und mobiles AT-System, das im Rahmen der Inklusion eingesetzt wird, beschreiben wir in diesem Artikel die Datenbrille „ADAMAAS“ (Adaptive and Mobile Action Assistance in Daily Living Activities). Das ADAMAAS-System ist mittels kognitiver Diagnostik und der Messung von Augenbewegungen in der Lage, individuelle Handlungsprobleme $\mathrm{zu}$ identifizieren, um gezielt nutzer- und handlungsspezifische Unterstützung zu leisten. Auf diese Weise kann die Datenbrille über die gezielte Einblendung von situationsabgestimmten Hilfskommentare und Hinweisen neue und zielgerichtete Unterstützungsoptionen für ein selbstbestimmtes Leben eröffnen und Nutzer mit Einschränkungen bedarfsgerecht und zielgerichtet in Alltagssituationen unterstützen.

\section{Assistive Technologien und Inklusion}

Die rasanten technischen Fortschritte in der Informations- und Kommunikationstechnologie (IKT) führen zur Entwicklung von immer neueren und leistungsstärkeren Assistiven Technologien (AT), die aktuell weite Bereiche unserer Berufs- und Alltagswelt durchdringen. Auch wenn es in der einschlägigen Literatur bis heute keine ein-

1 Im Folgenden wird aus Gründen der sprachlichen Vereinfachung nur die männliche Form verwendet. Es sind jedoch stets Personen männlichen und weiblichen Geschlechts gleichermaßen gemeint. Ә Open Access. (C) 2017 Essig, Strenge, Schack, publiziert von De Gruyter. (cc) BY-NC-ND Dieses Werk ist lizenziert unter der Creative Commons Attribution-NonCommercial-NoDerivatives 4.0 Lizenz. https://doi.org/10.1515/9783110561371-004 
deutige, allgemeingültige Definition von AT gibt, so versteht man darunter technische Hilfsmittel, die zu einer Aufrechterhaltung und/oder Verbesserung funktionaler Ressourcen eines Menschen führen und Einschränkungen ausgleichen, wobei der Fokus auf unterschiedlichen (Einsatz-)Schwerpunkten liegt. So werden bei der Definition von AT-Systemen Umgebungsveränderungen, Dienstleistungen und Prozesse berücksichtigt, die den Einsatz solcher Systeme durch Personen mit Einschränkungen und Ältere ermöglichen und diese in die Lage versetzen, an sozialen, politischen und ökonomischen Tätigkeiten teilzunehmen (U.S. Congress 1998; World Health Organization 2001). Assistierende Technologien können also Menschen unterstützen, die Schwierigkeiten beim Sprechen, Schreiben, Erinnern, Sehen, Hören und Lernen haben. Unterschiedliche Einschränkungen und Fähigkeiten erfordern dementsprechend den Einsatz unterschiedlicher AT-Systeme, da diese vorzugsweise auf ein spezielles Anwendungsgebiet zugeschnitten sind (Cook 2015).

Technische Geräte werden nicht nur zunehmend miteinander vernetzt, sondern es werden auch immer komplexere Interaktionsschnittstellen geschaffen. Das betrifft nicht nur die Steuerung von Maschinen in der Arbeitswelt, sondern auch den Einsatz von AT-Systemen in der beruflichen und privaten Welt, wie z. B. bei der Unterstützung von älteren Menschen, Menschen mit Behinderungen, oder Kindern mit Lernschwächen, um ihnen ein selbstbestimmtes Lernen und die Teilhabe an den gesellschaftlichen Entwicklungen zu ermöglichen. All dies zeigt, dass ein souveräner Umgang mit neuen Technologien immer mehr zur Grundkompetenz für Teilhabe und Gestaltungsmöglichkeiten in der demokratischen Gesellschaft wird (Bosse 2012). Die Zugänglichkeit zu diesen neuen Techniken stellt sich dabei aber gerade für Menschen mit Einschränkungen als Partizipationschance, aber auch als gesellschaftliches Exklusionsrisiko dar (Antener 2015), da diese Personen tendenziell über einen eingeschränkteren Zugang zu modernen Technologien und Medien verfügen. Beispielsweise sind für diese Zielgruppen, aufgrund von Problemen mit der Navigation und Schriftsprache, im Internet verfügbare Informationen oder Nutzungsmöglichkeiten schwieriger zu erschließen und die Angebote daher schlechter nutzbar: „Medienkompetenz als Bildungsauftrag wird - z. B. bei geistiger Behinderung - nicht in dem Maße wie bei nicht behinderten Menschen eingefordert“ (Lutz 2003: 148). Andererseits bieten moderne Technologien für diese Zielgruppen auch viele Chancen. Sie können Handlungsspielräume eröffnen, die Kommunikationsformen und -fähigkeiten erweitern, um auf diese Weise soziale Abhängigkeiten zu verringern und die Lebensqualität zu verbessern. Dabei bieten neue Technologien gerade für diese Bevölkerungsgruppen die Möglichkeit, sich besser mitteilen und sich dadurch viel stärker als bisher in der öffentlichen Wahrnehmung verankern zu können (Schaumburg 2010). So können Menschen mit Sprachstörungen beispielsweise mit den Kommunikationshilfen GoTalk9+ oder GoTalk20++ ${ }^{2}$ Sätze über das Antippen von Symbolen erzeugen

2 www.prentke-romich.de 
und sich hiermit der Umwelt mitteilen. Weiterhin spielt in virtuellen Räumen das Aussehen keine Rolle und es können soziale Kontakte unabhängig von räumlichen Barrieren gepflegt werden. Personen aus den Zielgruppen, die über Kompetenzen in der Anwendung und Nutzung von neuen Technologien verfügen, können besser in den ersten Arbeitsmarkt integriert werden und AT-Systeme erhöhen die Möglichkeit aktiv, selbstbestimmt und partizipativ ihre Freizeit zu gestalten (Bosse 2012; 2013).

AT in Form von Smart Glasses bieten hierbei große Chancen, da sie mobil einsetzbar sind, die Nutzer bei deren Anwendung beide Hände frei haben, und die Glasses wieder absetzen können, wenn keine Unterstützung mehr benötigt wird. Bisherige Anwendungen werden aktuell vor allem für die Unterstützung von ausgewählten Arbeitsabläufen bei industriellen Tätigkeiten oder bei der Gerätewartung vor Ort entwickelt (z. B. für Arbeit 4.0 oder Industrie 4.0). Dagegen existieren nur wenige Ansätze, die für den Bereich der Inklusion oder für hilfsbedürftige Menschen in Alltagssituationen entwickelt werden. In diesen Bereichen sind die Anforderungen an Smart Glasses besonders hoch: Zum einen müssen diese situations- und nutzerabhängig handeln (Diagnostik), sich individuell den sprachlichen, körperlichen und geistigen Einschränkungen anpassen, sowie eine an die jeweiligen Bedürfnisse genau abgestimmte Handlungsunterstützung bereitstellen, um den Nutzern ein selbstbestimmtes Leben, Lernen und Arbeiten zu ermöglichen. Weiterhin sind die Anwendungsszenarien in Alltagssituationen weniger deterministisch und standardisiert als industrielle Arbeitsabläufe. Daher müssen bei der Gestaltung von mobilen und kognitiven AT-Systemen für die Handlungsunterstützung in Alltagssituationen zahlreiche Komponenten zielgerichtet entwickelt und integriert werden. Im Folgenden wird anhand des ADAMAAS-Systems (Adaptive and Mobile Action Assistance; Essig 2016) veranschaulicht, welche (sozio-)technischen Aspekte hierbei zu berücksichtigen sind.

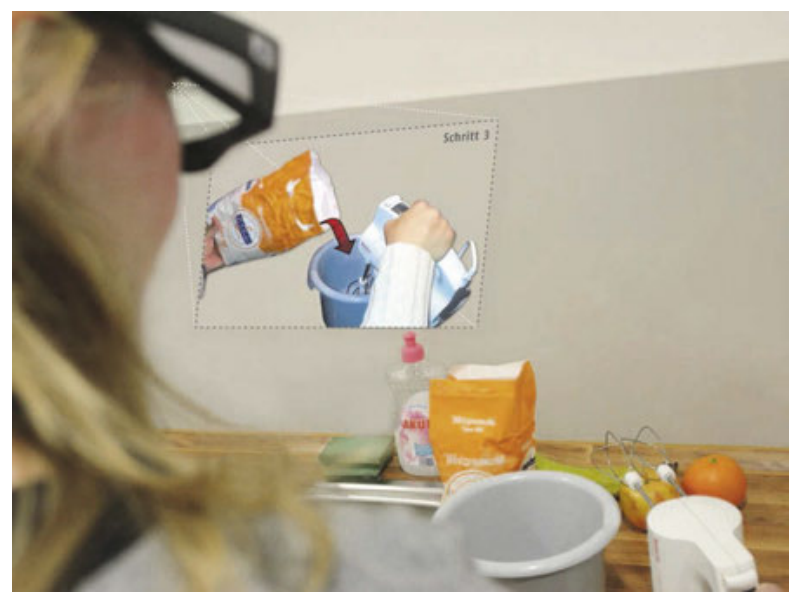

Abb. 1: Die ADAMAAS-Brille gibt situations-, nutzer- und handlungsspezifische Unterstützung auf einer transparenten virtuellen Ebene im Gesichtsfeld des Nutzers. 


\section{Die intelligente ADAMAAS-Datenbrille}

Das Projekt ADAMAAS beschäftigt sich mit der Entwicklung und Evaluation einer kognitiven und mit-handelnden Brille für die kontextsensitive und individualisierte Handlungsunterstützung für Menschen mit Einschränkungen und älteren Menschen in Alltagssituationen. Dabei werden Techniken aus den Bereichen Blickbewegungsmessung (Eyetracking), Objekt- und Handlungserkennung (Computer Vision) und Erweiterter Realität (Augmented Reality, AR) mit Techniken aus der Gedächtnisforschung und der Erfassung von Vitalparametern (z. B. Pulsveränderungen) kombiniert. In der AR werden Objekte der realen Welt mit virtuellen Informationen angereichert (siehe Abb. 1 und Abb. 2). Konkret werden Zusatzinformationen, in Form von textuellen, visuellen oder avatar basierten Hilfsinformationen auf einer transparenten virtuellen Ebene in das Sichtfeld des Nutzers eingeblendet und bei Kopf- und Körperbewegungen kontinuierlich angepasst (Wiesend 2016). Damit ist die ADAMAAS-Datenbrille nicht nur in der Lage sich individualisiert auf den jeweiligen Nutzer einzustellen, sondern auch die aktuellen Handlungsschritte zu erkennen, um eine gezielte und kontextabhängige Unterstützung zu geben. Im Folgenden werden die einzelnen Komponenten des ADAMAAS-Systems genauer beschrieben.

\subsection{ADAMAAS-Hardware}

Die ADAMAAS-Hardware besteht aus der ersten Eyetracking-Integration für Augmented Reality (AR), die vom Projektpartner Sensomotoric Instruments (SMI) ${ }^{3}$ entwickelt wurde. Dabei wurde das Epson Moverio BT-200 Head Mounted Display ${ }^{4}$ mit einer mobilen binokularen Eyetracking Plattform von SMI kombiniert. Auf diese Weise können Blickbewegungen nicht nur in der realen Welt, sondern auch auf einer virtuellen transparenten Ebene im Gesichtsfeld des Nutzers gemessen werden. Über die kontinuierliche Messung der Blickbewegungen kann bei Bedarf der Inhalt auf der AREbene dynamisch verändert werden. Das System besitzt eine Abtastrate von $30 \mathrm{~Hz}$ mit einer Genauigkeit von $<0.6^{\circ}$ auf dem AR-Display und ca. $0.5^{\circ}$ sonst. Die Epson Moverio BT-200 besitzt ein 0.42 Zoll LCD Display mit einer Auflösung von 960 x 540 Pixel, einem Sichtfeld von $23^{\circ}$ und einer Projektionsgröße von 40 Zoll in einer Distanz von 2,5m. Die ADAMAAS-Brille wiegt $110 \mathrm{~g}$.

3 www.smivision.com

4 www.epson.de 


\subsection{Messung Mentaler Repräsentationsstrukturen und Diagnostik}

Um eine altersgerechte, individuelle Assistenz in Alltagssituationen zu ermöglichen, werden in einem ersten Schritt kognitive Handlungsmuster des jeweiligen Nutzers diagnostiziert. Die dazu genutzte Structural-Dimensional Analysis Motoric (SDA-M)Methode (Schack 2012) ist in der kognitiven Psychologie etabliert und erfasst Gedächtnisstrukturen über eine hierarchische Zuordnungs-Prozedur (Splitting). Dadurch ist das System in der Lage, individuelle Probleme bei aktuellen Handlungsprozessen zu identifizieren und in Echtzeit durch die Einblendung entsprechender situationsspezifischer Hilfskommentare und Handlungshinweise auf einer transparenten Ebene im Gesichtsfeld des Nutzers die eigenständige Kontrolle von Alltagshandlungen $\mathrm{zu}$ erleichtern. Weiterhin können gezielt Lernprozesse induziert, Aufmerksamkeit gesteuert und die Aneignung neuer Handlungsmuster und -optionen unterstützt werden.

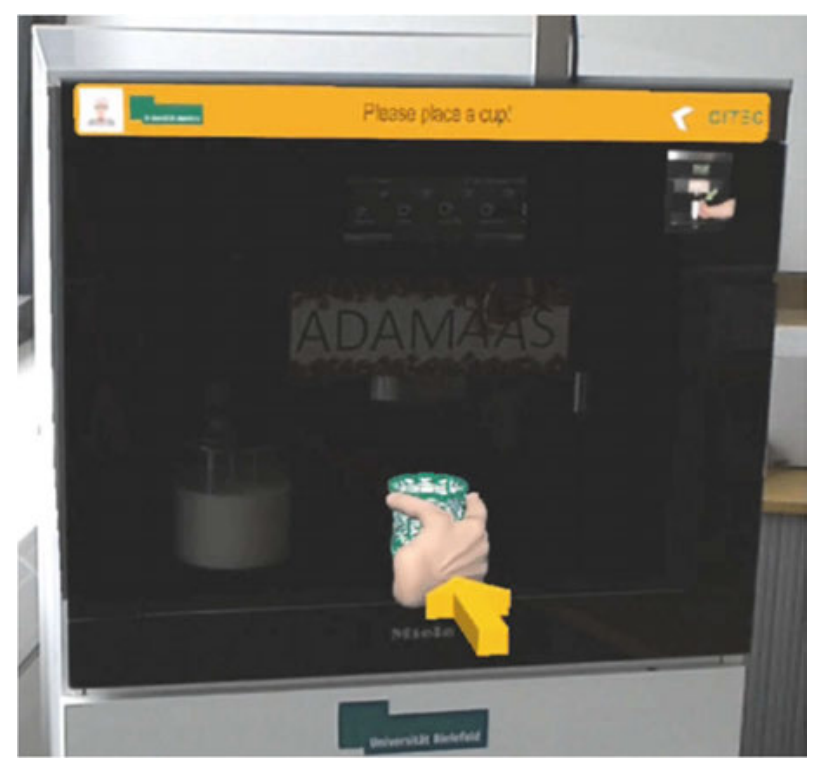

Abb. 2: Die AR-Animation zeigt den Nutzern, wie eine Tasse korrekt unter die Maschine gestellt wird.

\subsection{Objekt- und Handlungserkennung}

Um kontextabhängige Unterstützung anbieten zu können, muss das System in der Lage sein, automatisch Objekte und Handlungen $\mathrm{zu}$ erkennen und $\mathrm{zu}$ verfolgen 
(siehe Abb. 3). Im ADAMAAS-System geschieht dieses über Bildverarbeitung und technische Lernverfahren (z. B. Maschinelles Lernen oder Neuronale Netze) (Schroeder 2017). Darüber kann das System eigenständig Objekte und Handlungsschritte erkennen und darauf aufbauend entsprechende Handlungsunterstützung direkt auf das Display der Brille übertragen. So ist es beispielsweise möglich den Nutzer darauf hinzuweisen, wenn er ein falsches Objekt greift oder es in einer unkorrekten Weise bewegt oder anlegt.

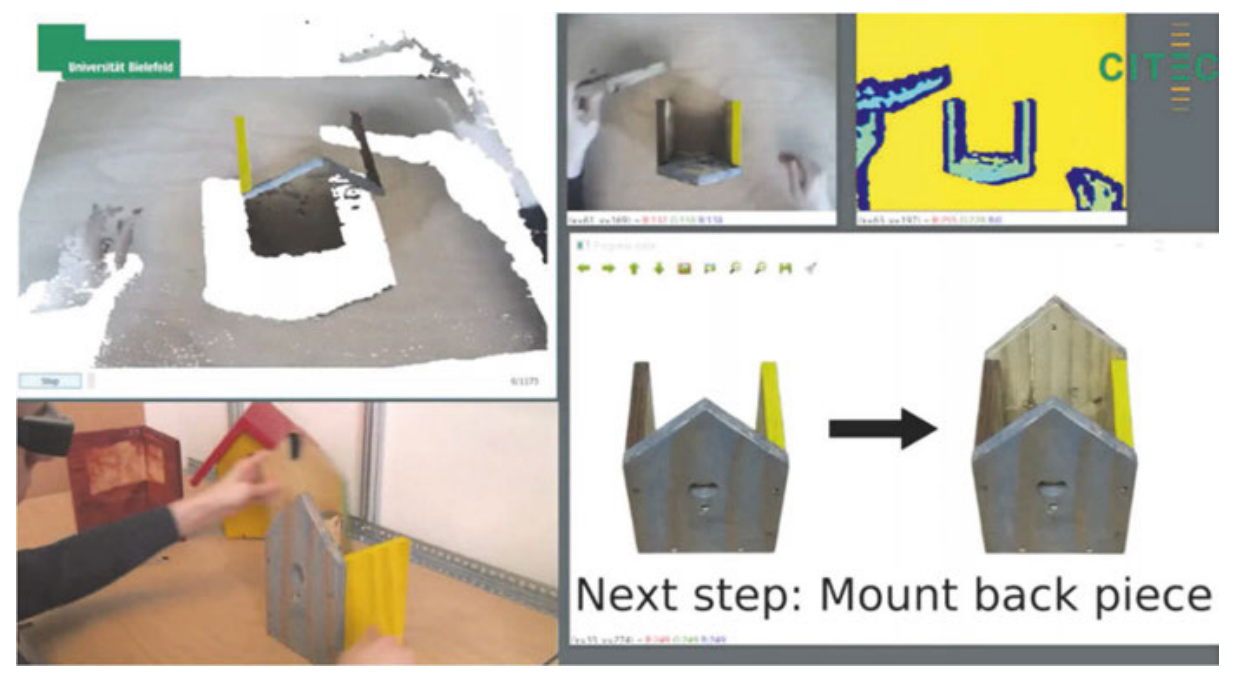

Abb. 3: Automatische Erkennung der Objekte und der Handlungssequenzen beim Zusammenbau eines Vogelhauses. Im Fenster unten rechts wird auf Basis des automatisch erkannten Zustandes (linke Seite) der darauf folgende Arbeitsschritt angezeigt.

\subsection{Augmented Reality und Sensordaten}

Die AR-Komponente (siehe Abb. 1 und Abb. 2) erlaubt die Darstellung von adaptivem Feedback in textueller, visueller oder Avatar basierter Form auf einer transparenten Ebene im Sichtfeld des Nutzers. Dabei erlaubt ein Marker basierter Ansatz die ADAMAAS-Datenbrille in der realen Welt zu verankern und das AR Feedback bei Kopfbewegungen entsprechend anzupassen. Als Hinweise dienen nicht nur Bilder, die mittels Blickbewegungen vergrößert werden können, sondern auch Animationen und Videoüberlagerungen. Auch Sensordaten (wie z. B. die Herzfrequenz oder die Oberkörperneigung), die über einen Brustsensor gemessen werden, können in das Feedback mit eingebunden werden. 


\section{Zusammenfassung}

In diesem Artikel werden anhand des ADAMAAS-Projekts Wege aufgezeigt, wie Smart Glasses über den Einsatz von kognitiven Methoden dahingehend erweitert werden können, eine individualisierte und kontextabhängige Inklusionsunterstützung für unterschiedliche Zielgruppen in verschiedensten Alltagsanwendungen zu geben. Dabei geht es darum, modulare Techniken und Methoden zu entwickeln und umzusetzen, die sukzessive an unterschiedliche Anwendungsszenarien und -kontexte, sowie an unterschiedliche Nutzeranforderungen angepasst werden können, um ein selbstbestimmtes Leben zu ermöglichen. Dabei ist es wichtig, über User Experience Studien die Zielgruppen bereits frühzeitig in den Entwicklungsprozess mit einzubeziehen und entsprechende Hinweise für Optimierungskriterien zu bekommen. Des Weiteren sind Datenschutz, sowie ethische, rechtliche, sicherheitsrelevante und soziale Fragestellungen (ELSI-Aspekte) zu berücksichtigen (Manzeschke 2013). Noch sind die Hardware Eigenschaften der Smart Glasses nicht ausreichend, um Systeme umzusetzen, die eine inobstrusive Unterstützung bei langen Benutzungszeiten erlauben. Doch gehen die Entwicklungsanstrengungen in die Richtung Smart Glasses zu entwickeln, die sich immer mehr den normalen Sehhilfen angleichen. Daher ist es wichtig bereits heute an der Umsetzung von neuen Techniken und Methoden zu arbeiten, um bei der Einführung von verbesserter Hardware schnell neue Wege in der Inklusion einschlagen zu können.

\section{Dank}

Die Forschung im Rahmen des Projektes ADAMAAS wird vom Bundesministerium für Bildung und Forschung (BMBF) finanziert. Die Arbeit wird außerdem vom Exzellenzcluster „Kognitive Interaktionstechnologie ,CITEC““ (EXC 277) unterstützt, der von der Deutschen Forschungsgemeinschaft (DFG) gefördert wird. 


\section{Literaturverzeichnis}

Antener, G. (2015): Einfach surfen? Internetzugang für Menschen mit geistiger Behinderung. Mediale Welt inklusive! Sichtbarkeit und Teilhabe von Menschen mit Behinderungen in den Medien, Seismo, Zürich.

Bosse, Ingo (2012): Medienbildung im Zeitalter der Inklusion, 1st Edition, Landesanstalt für Medien Nordrhein-Westfalen (LFM), Düsseldorf, Germany.

Bosse, Ingo (2013): Keine Bildung ohne Medien! Perspektiven der Geistigbehindertenpädagogik, 1st Edition, Teilhabe Berlin, Germany, S. 26-32.

Capovilla, Dino und Markus Gebhardt (2016): Assistive Technologien für Menschen mit Sehschädigung im inklusiven Unterricht. Zeitschrift für Heilpädagogik, 1(1), S. 4-15.

Cook, Albert M. und Janice Miller Polgar (2015): Assistive Technologies: Principles and Practice, 4th Revision. Mosby: Maryland Heights, Missouri, USA.

Essig, Kai, Strenge, Benjamin und Thomas Schack (2016): ADAMAAS - Towards Smart Glasses for Mobile and Personalized Action Assistance. 9th International Conference on Pervasive Technologies Related to Assistive Environments (PETRA'16), June 29th - July 1st, Corfu, Greece,

Lutz, Klaus (2003): Medienarbeit mit Behinderten: medien+erziehung, 3, München, Germany, S. 148-151.

Manzeschke, Arne, Weber, Karsten, Rother, Elisabeth und Heiner Fangerau (2013): Ergebnisse der Studie »Ethische Fragen im Bereich Altersgerechter Assistenzsysteme. Berlin:VDI/VDE Innovation + Technik.

Nierling, Linda and Maia, João Maria, Hennen, Leonhard und Gregor Wolbring (2017): Assistive Technologien für die Inklusion von Menschen mit Behinderungen in Gesellschaft, Bildung und Arbeitsmarkt. Fraunhofer-Institut für System- und Innovationsforschung (ISI), Institut für Technikfolgen-Abschätzung der ÖAW (ITA), RT Paris (URL= https://www.itas.kit.edu/projekte_nier16_asstech.php).

Schack, Thomas (2012): A method for measuring mental representation. In: Handbook of measurement in sport and exercise psychology. Champaign, IL: Human Kinetics, S. 231-252.

Schaumburg, Melanie (2010): Medienpädagogik mit Menschen mit geistiger Behinderung - Eine methodische Anleitung für die Praxis, 1 (1), Heilpädagogik Online, S. 5-19.

Schroeder, Matthias und Helge Ritter (2017): Deep Learning for Action Recognition in Augmented Reality Assistance Systems. ACM SIGGRAPH 2017. Champaign, IL: Human Kinetics\}, S. 231-252.

U.S. CONGRESS: Assistive Technology Act of 1998 (1998): URL: https://www.congress.gov/bill/105th-congress/senate-bill/2432/text.

Wiesend, Stephan (2016): AR- \& VR-Lösungen in Unternehmen. Computer Woche, URL: http://www.computerwoche.de/a/ar-und-vr-loesungen-im-unternehmen,3217983,2, Last access: 21.08.2016.

WHO - World Health Organisation (2016): Weltbericht über Altern und Gesundheit. URL: http://apps.who.int/iris/bitstream/10665/186468/20/WHO_FWC_ALC_15.01_ger.pdf. 